\title{
1-METILCICLOPROPENO (1-MCP) ACUOSO PARA RETRASAR MADURACIÓN EN FRUTOS DE MANGO KEITT CON TRATAMIENTO HIDROTÉRMICO CUARENTENARIO
}

\author{
AQUEOUS 1-METHYLCYCLOPROPENE (1-MCP) TO DELAY RIPENING OF \\ KEITT MANGO FRUIT WITH QUARANTINE HOT WATER TREATMENT
}

\author{
Jorge A. Osuna-García*, Yolanda Nolasco-González, \\ Ma. Hilda Pérez-Barraza, Rafael Gómez-Jaimes y Mario A. Urías-López
}

Campo Experimental Santiago Ixcuintla, Instituto Nacional de Investigaciones Forestales, Agrícolas y Pecuarias. Km. 6 Entronque Carretera Internacional México-Nogales. 63300, Santiago Ixcuintla, Nayarit. Tel (55) 38718700 Ext. 84415.

*Autor para correspondencia (osuna.jorgealberto@inifap.gob.mx)

\section{RESUMEN}

El 1-metilciclopropeno (1-MCP) en formulación gaseosa retrasa el proceso de maduración en algunas variedades de mango (Mangifera indica L.) pero requiere $12 \mathrm{~h}$ de aplicación en contenedores herméticos. Recientemente se ha desarrollado una formulación acuosa, la cual ha demostrado la misma efectividad que el 1-MCP en forma gaseosa al ser aplicada en postcosecha por inmersión de 1 a 5 min, lo que permite mayor flexibilidad para su aplicación. Los objetivos de esta investigación fueron determinar la efectividad del 1-MCP acuoso para retrasar el proceso de maduración, extender la vida de anaquel y mantener la calidad de frutos de mango var. Keitt, así como comprobar si ésta es afectada por el tratamiento hidrotérmico cuarentenario (THC). Se realizaron tres experimentos, dos durante 2013 en Nayarit, México y Florida, EUA y uno durante 2014 en Nayarit, México. La formulación acuosa del 1-MCP retrasó el proceso de maduración al mantener la firmeza por mayor tiempo, pero mostró una interacción negativa con el THC al causar manchas en la cáscara y oscurecimiento de lenticelas. El efecto negativo fue menor cuando el 1-MCP acuoso se aplicó antes o después del THC sin hidroenfriado y mayor cuando se aplicó después del THC + hidroenfriado. En contraste, el 1-MCP acuoso aplicado a frutos sin THC mantuvo $92 \%$ de la firmeza inicial al término de los 21 d de simulación de traslado refrigerado y retrasó hasta 4 d el proceso de maduración durante la simulación de mercadeo sin afectar la apariencia externa, por lo que puede ser una alternativa viable para mercados que no demandan el THC obligatorio.

Palabras clave: Mangifera indica, 1-MCP acuoso, calidad, color de pulpa, firmeza de fruto.

\section{SUMMARY}

Gaseous 1-methylcyclopropene (1-MCP) delays fruit ripening in some mango (Mangifera indica L.) varieties, but it requires $12 \mathrm{~h}$ of application in sealed containers. A recently developed aqueous formulation has demonstrated the same effectiveness as gaseous 1-MCP when applied as a postharvest dip for 1 to $5 \mathrm{~min}$, allowing more flexibility for its application. The objectives of this research were to determine the effectiveness of aqueous 1-MCP on delaying the ripening process, extension of shelf life, and quality maintenance of Keitt mango fruits, as well as to prove whether the quality is affected by the Quarantine Hot Water Treatment (QHWT). Three experiments were conducted, two of them during 2013 in Nayarit, Mexico and Florida, USA and another one during 2014 in Nayarit, Mexico. Aqueous 1-MCP caused some delay of fruit ripening as shown by improved maintenance of fruit firmness, but it had a negative interaction with QHWT causing fruit spots and lenticel darkening. This negative effect was lower when 1-MCP was applied before or after QHWT without hydrocooling, and higher when applied after
QHWT + hydrocooling. In contrast, aqueous 1-MCP applied to fruits without QHWT maintained $92 \%$ of the initial firmness at the end of the 21-day period of refrigerated shipping simulation and delayed the ripening process for 4 $\mathrm{d}$ during marketing simulation without affecting external appearance. Thus, aqueous 1-MCP may represent a viable alternative for markets that do not require mandatory $\mathrm{QHWT}$.

Index words: Mangifera indica, aqueous 1-MCP, quality, pulp color, fruit firmness.

\section{INTRODUCCIÓN}

El mango (Mangifera indica L.) es uno de los frutos con mayor volumen de importación en el mundo. Durante el año 2009 se comercializaron 860,000 t con un valor de 1,012 millones de dólares (FAOSTAT, 2011). Los principales países importadores son Estados Unidos con $31 \%$ del total y algunos de la Unión Europea que contabilizan el 19 $\%$ del total. Con muy pocas excepciones, Estados Unidos demanda tratamiento hidrotérmico cuarentenario (THC) obligatorio para la totalidad de sus mangos importados, en tanto que el tratamiento no es imperativo para los países de la Unión Europea.

El 1-MCP es un potente inhibidor del etileno que previene su efecto en varias frutas, hortalizas y flores. Según la especie, el 1-MCP afecta la respiración, la producción de etileno, la producción de compuestos volátiles, la degradación de clorofilas y la síntesis de pigmentos, así como cambios en proteínas y polisacáridos de la pared celular, presencia de enfermedades y desórdenes fisiológicos, además de afectaciones en la acidez y contenido de azúcares (Watkins, 2006 y 2008).

La utilidad del 1-MCP se ha probado en variedades de mango como Keitt (Osuna, 2006), Kent (Osuna et al., 2005a; Osuna-García et al., 2009), Nam Dokmai (Penchaiya et al., 2006), Namh-dawg-mai-sri-tong (Chaiprasart y Hansawasdi, 2009), Ataulfo (Muy et al., 2009; Ortiz-Franco 
et al., 2016) y Tommy Atkins (Coêlho et al., 2006; Pereira et al., 2011). En la mayoría de los experimentos se utilizó el 1-MCP gaseoso en cámaras herméticas con dosis desde 100 hasta 1200 partes por billón (ppb) aplicado por 120 $24 \mathrm{~h}$ a temperatura ambiente (de 22 a $25^{\circ} \mathrm{C}$; HR de 70 a $80 \%$ ) o mientras se enfriaba la fruta a $12{ }^{\circ} \mathrm{C}$ con $90 \%$ de HR. Los estudios mostraron que el 1-MCP retrasó el pico climatérico y disminuyó la producción de etileno, la firmeza de pulpa se mantuvo por más tiempo y se retrasaron los cambios de color de pulpa.

La adopción del 1-MCP a nivel comercial ha sido limitada, en parte por el tiempo de aplicación y su eficacia puede afectarse por el tratamiento cuarentenario con agua caliente. Osuna-García et al. (2007) evaluaron el efecto de 1-MCP a 300 ppb después del tratamiento con agua caliente $\left[52^{\circ} \mathrm{C}\right.$ por 5 min (tratamiento para antracnosis) y $46.1^{\circ} \mathrm{C}$ por 110 min (tratamiento cuarentenario para control de larva de mosca de la fruta)] sobre la fisiología y la calidad del mango Keitt y encontraron que la acción del 1-MCP se redujo por el grado de tratamiento con agua caliente; a mayor tiempo de tratamiento hidrotérmico, menor eficacia del 1-MCP. Recientemente Ngamchuachit et al. (2014) indicaron que la aplicación del 1-MCP antes del THC redujo la tasa de ablandamiento del fruto en comparación con la aplicación del 1-MCP después del THC.

La formulación acuosa de 1-MCP es una alternativa de mayor flexibilidad de aplicación en pre y postcosecha. En frutos de mango, aguacate (Persea americana Mill.), tomate (Solanum lycopersicum), ciruela (Prunus salicina) y pera (Pyrus communis), la aplicación postcosecha por inmersión durante 1 a 5 min mostró la misma eficacia que la aplicación de 9 a 12 h del 1-MCP gaseoso, al retrasar el proceso de maduración y el ablandamiento (Cheng et al., 2012; Choi et al., 2008; Choi y Huber, 2008; Manganaris et al., 2008). Esta formulación podría ser fácilmente incorporada en los procesos de empaque de mango, ya sea después de lavar la fruta o después del tratamiento con agua caliente. De ser positiva, constituiría una excelente alternativa ya que reduciría significativamente el tiempo de aplicación y sería fácilmente adoptada por los empacadores.

Los objetivos de esta investigación fueron determinar la efectividad del 1-MCP acuoso para retrasar el proceso de maduración, extender vida de anaquel y mantener calidad de frutos de mango Keitt, así como comprobar si ésta es afectada por el THC.

\section{MATERIALES Y MÉTODOS}

Se realizaron tres experimentos; dos en 2013 y uno en 2014. El primer experimento se realizó en Nayarit, México, identificado como Temporada 2013 Nayarit y el segundo en Florida, USA, referido como Temporada 2013 Florida, para corroborar resultados con frutos de otra región y que se sometieron a THC a nivel planta piloto de laboratorio. El tercer experimento se realizó en 2014, nuevamente en Nayarit, México en el que se consideró todo lo observado en 2013 para el establecimiento de los tratamientos y entender el efecto de los factores involucrados en los experimentos sobre la acción del 1-MCP en la calidad de los frutos.

\section{Experimento 1. Temporada 2013 Nayarit}

En julio de 2013 se obtuvieron frutos de mango Keitt de una empacadora comercial de exportación. Se seleccionaron 70 frutos por tratamiento con peso de 501 a $700 \mathrm{~g}$, en madurez fisiológica, libres de daños mecánicos, plagas y enfermedades, mismos que se sometieron a los siguientes tratamientos: 1) testigo absoluto (sin THC; $\sin 1-\mathrm{MCP} ; \sin$ hidroenfriado); 2) testigo 1-MCP (con 1-MCP pero sin THC e hidroenfriado); 3) testigo THC (sólo THC sin hidroenfriado); 4) 1-MCP antes de THC sin hidroenfriado; 5) 1-MCP después de THC sin hidroenfriado, y 6) 1-MCP después de $\mathrm{THC}+$ hidroenfriado.

El 1-MCP acuoso (AF×RD-038; $3.8 \%$ 1-MCP, $\leq 5 \%$ dextrosa, $88-95 \%$ ciclodextrina y $1-5 \%$ material inerte) se preparó a dosis de $625 \mathrm{\mu g} \mathrm{L}^{-1}$ en $200 \mathrm{~L}$ de agua de la llave en contenedores plásticos de $512 \mathrm{~L}(0.80 \times 0.80 \times 0.80 \mathrm{~m})$ y se aplicó en los primeros 20 min después de su preparación. Los frutos fueron sumergidos durante 5 min antes o después del THC. El THC fue el contemplado por la norma del Departamento de Agricultura de los Estados Unidos (USDA, por sus siglas en inglés) $\left(46.1^{\circ} \mathrm{C}\right.$ por $\left.90 \mathrm{~min}\right)$ e hidroenfriado de 21 a $23{ }^{\circ} \mathrm{C}$ por $30 \mathrm{~min}$. Posteriormente, los frutos fueron trasladados al laboratorio de postcosecha del Instituto Nacional de Investigaciones Forestales, Agrícolas y Pecuarias (INIFAP, Campo Experimental Santiago Ixcuintla) para su almacenamiento en refrigeración (12 \pm 1 ${ }^{\circ} \mathrm{C}$; $90 \pm 5 \% \mathrm{HR}$ ) por $21 \mathrm{~d}$ y posterior simulación de mercadeo $\left(22 \pm 2{ }^{\circ} \mathrm{C} ; 75 \pm 10 \% \mathrm{HR}\right)$ hasta madurez de consumo. Los muestreos se realizaron al inicio y al final del almacenamiento refrigerado y después en los días 4 y 7 de simulación de mercadeo. Se utilizó un diseño completamente al azar con 20 frutos para pérdida de peso y ocho para el resto de variables. Se realizó análisis de varianza mediante el procedimiento GLM del SAS y pruebas de comparación de medias (Duncan, 0.05) (SAS Institute, 2002).

\section{Experimento 2. Temporada 2013 Florida}

Se utilizaron frutos de la variedad Keitt cosechados en madurez fisiológica del Banco de Germoplasma del USDA-Agricultural Research Service (ARS) en Miami, Florida en agosto de 2013. Los frutos (40 por tratamiento), se 
trasladaron el mismo día al Laboratorio de Postcosecha del Departamento de Horticultura en la Universidad de Florida. Al día siguiente se lavaron con una solución detergente (Dawn Ultra' ${ }^{\mathrm{T}} ; 1 \mathrm{~mL} \mathrm{~L}^{-1}$ ), se secaron con toallas absorbentes, se seleccionaron por tamaño uniforme y ausencia de daños mecánicos, plagas y enfermedades, y se sometieron a los siguientes tratamientos: 1) testigo hidrotérmico (sÓlo THC + hidroenfriado); 2) 1-MCP después de THC sin hidroenfriado; 3) 1-MCP antes de THC sin hidroenfriado y, 4) 1-MCP después de THC + hidroenfriado. Se utilizó la formulación acuosa del 1-MCP (AFxRD-038) a dosis de $625 \mathrm{\mu g} \mathrm{L}^{-1}$ disuelta en $50 \mathrm{~L}$ de agua destilada en contenedores plásticos de $90 \mathrm{~L}$ de capacidad en los que se sumergieron los frutos por $5 \mathrm{~min}$.

El THC y el hidroenfriado se realizaron de acuerdo con la norma de USDA pero en tinas experimentales (modelo $\mathrm{HWH}-2$, Gaffney Eng., Gainesville, FL, USA). Enseguida se realizó el muestreo inicial y el resto de los frutos se mantuvo en refrigeración $\left(12 \pm 1^{\circ} \mathrm{C}\right.$; $\left.90 \pm 5 \% \mathrm{HR}\right)$ por $21 \mathrm{~d}$, y luego en simulación de mercadeo $\left(22 \pm 2{ }^{\circ} \mathrm{C} ; 75 \pm 10 \% \mathrm{HR}\right)$ hasta madurez de consumo. Los muestreos se realizaron al inicio y al final del almacenamiento refrigerado y a madurez de consumo. Se utilizó un diseño completamente al azar con 10 frutos para pérdida de peso y cinco para el resto de variables. Se realizó análisis de varianza mediante el procedimiento GLM del SAS y pruebas de comparación de medias (Duncan, 0.05) (SAS Institute, 2002).

\section{Experimento 3. Temporada 2014 Nayarit}

En julio de 2014 se colectaron frutos de la variedad Keitt de una empacadora comercial de mango para exportación con características similares a los experimentos previos con los cuales se formaron dos grupos: a) frutos con THC e hidroenfriado y b) frutos sin THC ni hidroenfriado. El THC fue el contemplado en la norma del USDA $\left(46.1^{\circ} \mathrm{C}\right.$ por 90 min) e hidroenfriado de 21 a $23^{\circ} \mathrm{C}$ por $30 \mathrm{~min}$. El 1-MCP acuoso (AF×RD-038) a dosis de 0, 400, 800 y $1200 \mu \mathrm{g} \mathrm{L}^{-1}$ en agua de la llave fue aplicado por inmersión por 3 min simultáneamente a frutos con o sin THC. Después de la aplicación del 1-MCP los frutos que tendrían THC se sometieron a las condiciones especificadas por la norma, en tanto que los que no llevaron THC se mantuvieron bajo condiciones ambientales.

Al término de los tratamientos ambos grupos se trasladaron al laboratorio de postcosecha del INIFAP-Campo Experimental Santiago Ixcuintla para su análisis inicial y conservación en refrigeración $\left(12 \pm 1^{\circ} \mathrm{C}\right.$; $\left.90 \pm 5 \% \mathrm{HR}\right)$ por $21 \mathrm{~d}$ y posterior simulación de mercadeo $\left(22 \pm 2^{\circ} \mathrm{C} ; 75 \pm 10\right.$ $\% \mathrm{HR}$ ) hasta madurez de consumo. Los muestreos se realizaron al inicio y al final del almacenamiento refrigerado y después en los días 4 y 7 de simulación de mercadeo. Se utilizó un diseño completamente al azar con arreglo factorial en el que se consideraron: el Factor A (con o $\sin \mathrm{THC}$ ) y el Factor B (las dosis de 1-MCP) con 10 frutos para pérdida de peso y cinco para el resto de variables. Se realizó análisis de varianza mediante el procedimiento GLM del SAS y prueba de comparación de medias (Duncan, 0.05) (SAS Institute, 2002).

Las variables evaluadas en los tres experimentos fueron pérdida de masa (PM), apariencia externa, firmeza de pulpa, color de pulpa y sólidos solubles totales. La pérdida de masa se determinó mediante una báscula digital portátil con capacidad de $4800 \mathrm{~g}$ y aproximación de $0.1 \mathrm{~g}$ (Acculab $\circledast$ modelo VI-4800, Florham Park, NJ, USA). Se usaron 20 frutos para registro de peso y cálculo de la pérdida acumulada en porcentaje. La apariencia externa se calificó con base en una escala visual donde 0 = excelente; 1 = buena; 2 = regular y 3 = mala. Los límites comerciales para apariencia externa se ubican en valores de 0 a 1. La firmeza se determinó con un penetrómetro Chatillon $\AA$ modelo DFE-050 (Ametek Instruments, Largo, FL, USA), con punzón cilíndrico de $10 \mathrm{~mm}$ de diámetro; las medidas se tomaron en la región ecuatorial del fruto entero. Una sección de $5 \mathrm{~mm}$ de la cáscara se removió para exponer la pulpa y el punzón se insertó a $4 \mathrm{~mm}$ de profundidad a una velocidad de $180 \mathrm{~mm} \mathrm{~min}^{-1}$. Los datos se expresaron en Newtons (N). El color de pulpa se midió con un colorímetro Konica Minolta modelo CR-400 (Konica Minolta Sensing Americas, Inc., Ramsey, NJ, USA) con iluminación estándar C. Las medidas se realizaron a la mitad del fruto y la pulpa estuvo expuesta, aproximadamente, a $1 \mathrm{~cm}$ paralelo a la semilla. Los datos se expresaron en ángulo de tono ('hue). Los sólidos solubles totales fueron determinados mediante un refractómetro digital con compensador automático de temperatura Atago ${ }^{\circledR}$ modelo PAL-1 (Atago Inc., Bellewua, WA, USA) calibrado con agua destilada (AOAC, 1984).

\section{RESULTADOS Y DISCUSIÓN}

\section{Pérdida de masa (PM)}

El efecto del 1-MCP acuoso, a dosis de $625 \mathrm{\mu g} \mathrm{L}^{-1}$ aplicado por inmersión por 5 min sobre PM de frutos de mango Keitt, fue influenciado por el THC. En el experimento realizado en Nayarit durante 2013 (Cuadro 1A), al término del periodo de simulación de traslado refrigerado (21 d a $12 \pm$ $1^{\circ} \mathrm{C} ; 90 \pm 5 \% \mathrm{HR}$ ) el testigo absoluto y el testigo 1-MCP presentaron los menores valores de PM (2.3 y $2.6 \%$, respectivamente). En contraste, los tratamientos con 1-MCP, aplicados antes o después del THC, y el testigo con hidrotérmico presentaron pérdidas entre 3.4 y $3.7 \%$, respectivamente. El tratamiento con mayor PM fue el 1-MCP aplicado después del THC + hidroenfriado con $4.3 \%$. 
Durante la simulación de mercadeo al día 4 se formaron dos grupos estadísticamente diferentes, los frutos de los testigos absoluto y 1-MCP sin THC con pérdidas menores (4.7 a $5.1 \%$ ) y los que tenían THC, solo o en cualquier combinación con 1-MCP presentaron pérdidas de 6.6 a 7.1 $\%$. Esta tendencia se mantuvo hasta madurez de consumo donde el testigo absoluto y el testigo 1-MCP mostraron pérdidas entre 6.6 y $7.4 \%$, respectivamente, mientras que los frutos testigo del THC y los tratados con 1-MCP, aplicado antes o después del THC, presentaron pérdidas entre 8.9 y $9.4 \%$, respectivamente. En contraste, para el expe- rimento realizado en Florida (Cuadro 1B), al término de la simulación de traslado refrigerado, los frutos tratados con 1-MCP antes o después del THC mostraron menor PM (4.3 y $4.4 \%$, respectivamente) que aquéllos tratados sólo con THC o THC + hidroenfriado (6.1 y $6.6 \%$, respectivamente).

Cabe mencionar que los frutos de los tratamientos testigo y 1-MCP, aplicado después de THC + hidroenfriado, sólo llegaron hasta la etapa de término de refrigeración, y los tratados con 1-MCP, antes o después del THC, alcanzaron la madurez de consumo. Posiblemente esto sucedió

Cuadro 1. Pérdida de masa (\%) de frutos de mango Keitt tratados con 1-metilciclopropeno (1-MCP) acuoso con o sin tratamiento hidrotérmico cuarentenario (THC).

\section{A) Experimento 1. Nayarit 2013.}

\begin{tabular}{lcccc}
\hline \multirow{2}{*}{ Tratamiento } & \multicolumn{4}{c}{ Etapas de muestreo } \\
\cline { 2 - 5 } & Inicial & $21 \mathrm{DR}+0 \mathrm{Amb}^{+}$ & $21 \mathrm{DR}+4 \mathrm{Amb}$ & $21 \mathrm{DR}+7 \mathrm{Amb}$ \\
\hline 1. Testigo Absoluto & $0.0 \mathrm{a}$ & $2.3 \mathrm{c}$ & $4.7 \mathrm{~b}$ & $6.6 \mathrm{~b}$ \\
2. Testigo 1-MCP & $0.0 \mathrm{a}$ & $2.6 \mathrm{c}$ & $5.1 \mathrm{~b}$ & $7.4 \mathrm{~b}$ \\
3. Testigo THC & $0.0 \mathrm{a}$ & $3.4 \mathrm{~b}$ & $6.9 \mathrm{a}$ & $9.2 \mathrm{a}$ \\
4. 1-MCP antes THC & $0.0 \mathrm{a}$ & $3.7 \mathrm{~b}$ & $6.7 \mathrm{a}$ & $9.0 \mathrm{a}$ \\
5. 1-MCP después THC & $0.0 \mathrm{a}$ & $3.6 \mathrm{~b}$ & $6.6 \mathrm{a}$ & $8.9 \mathrm{a}$ \\
6. 1-MCP después THC + Hidro & $0.0 \mathrm{a}$ & $4.3 \mathrm{a}$ & $7.1 \mathrm{a}$ & $9.4 \mathrm{a}$ \\
\hline
\end{tabular}

Medias con letras iguales dentro de columnas no son estadísticamente diferentes (Duncan, 0.05).

B) Experimento 2. Florida 2013.

\begin{tabular}{lccc}
\hline Tratamiento & \multicolumn{3}{c}{ Etapas de muestreo } \\
\cline { 2 - 4 } & Inicial & $21 \mathrm{DR}+0 \mathrm{Amb}$ & $21 \mathrm{DR}+4 \mathrm{Amb}$ \\
\hline 1. Testigo & $0.0 \mathrm{a}$ & $6.1 \mathrm{a}$ & - \\
2. 1-MCP después THC & $0.0 \mathrm{a}$ & $4.3 \mathrm{~b}$ & $6.1 \mathrm{a}$ \\
3. 1-MCP antes THC & $0.0 \mathrm{a}$ & $4.4 \mathrm{~b}$ & $6.4 \mathrm{a}$ \\
4. 1-MCP después THC + Hidro & $0.0 \mathrm{a}$ & $6.6 \mathrm{a}$ & - \\
\hline
\end{tabular}

Medias con letras iguales dentro de columnas no son estadísticamente diferentes (Duncan, 0.05).

\section{C) Experimento 3. Nayarit 2014.}

\begin{tabular}{|c|c|c|c|c|}
\hline \multirow{2}{*}{ Tratamiento } & \multicolumn{4}{|c|}{ Etapas de muestreo } \\
\hline & Inicial & $21 \mathrm{DR}+0 \mathrm{Amb}$ & $21 \mathrm{DR}+4 \mathrm{Amb}$ & $21 \mathrm{DR}+7 \mathrm{Amb}$ \\
\hline & \multicolumn{4}{|c|}{ Factor $\mathrm{A}(\mathrm{THC})$} \\
\hline 1. $\operatorname{Sin}$ & $0.0 \mathrm{a}$ & $2.6 b$ & $4.4 \mathrm{~b}$ & $5.9 b$ \\
\hline \multirow[t]{2}{*}{ 2. Con } & $0.0 \mathrm{a}$ & $3.1 \mathrm{a}$ & $5.4 \mathrm{a}$ & $7.8 \mathrm{a}$ \\
\hline & \multicolumn{4}{|c|}{ Factor B (Dosis 1-MCP) } \\
\hline 1. $0 \mu \mathrm{g} \mathrm{L}^{-1}$ & $0.0 \mathrm{a}$ & $2.9 \mathrm{a}$ & $5.0 \mathrm{a}$ & $7.4 \mathrm{a}$ \\
\hline 2. $400 \mu \mathrm{g} \mathrm{L}^{-1}$ & $0.0 \mathrm{a}$ & $2.7 \mathrm{a}$ & $4.7 \mathrm{a}$ & $6.1 \mathrm{~b}$ \\
\hline 3. $800 \mu \mathrm{g} \mathrm{L}^{-1}$ & $0.0 \mathrm{a}$ & $2.9 \mathrm{a}$ & 4.9 a & $6.1 \mathrm{~b}$ \\
\hline 4. $1200 \mu \mathrm{g} \mathrm{L}^{-1}$ & $0.0 \mathrm{a}$ & $2.9 \mathrm{a}$ & 4.9 a & $6.6 \mathrm{ab}$ \\
\hline
\end{tabular}

Medias con letras iguales por factor dentro de columnas no son estadísticamente diferentes (Duncan, 0.05). ${ }^{+} \mathrm{DR}=\mathrm{d}$ refrigeración; Amb $=\mathrm{d}$ de simulación de mercadeo. 
debido a que los frutos fueron cosechados de un banco de germoplasma al cual no se le había realizado ninguna aplicación de fungicidas, además de haber sido sometidos a THC en las mismas tinas y agua en las que ya se había realizado el THC de los tratamientos de 1-MCP antes o después de THC. Es probable que la concentración de esporas de antracnosis presente en el agua para dichos tratamientos fuera demasiado alta, e impidiera que los frutos llegaran a madurez de consumo.

Debido a que en estos experimentos no se pudo precisar el efecto del THC o del 1-MCP, de manera individual o en combinación en las variables estudiadas, se decidió establecer un tercer experimento para separar el efecto de ambos factores durante la temporada 2014 en Nayarit. Se realizó el análisis de varianza y en ninguna de las variables se encontró diferencia significativa $(P \leq 0.05)$ para la interacción por lo cual se presentan sólo los efectos independientes de cada factor principal en estudio. Los resultados mostraron un claro efecto del THC (Cuadro 1C) ya que la PM observada, desde el término de simulación de traslado refrigerado hasta madurez de consumo para los frutos con THC, fue estadísticamente superior a la de los frutos sin THC con valores acumulados de 7.8 y $5.9 \%$, respectivamente. En contraste, el efecto del 1-MCP no fue tan claro sobre la PM ya que los frutos aplicados con las diferentes dosis de 1-MCP no presentaron diferencias estadísticas en comparación a los frutos testigo desde el inicio hasta 4 d de simulación de mercadeo y sólo en madurez de consumo los frutos aplicados con 400 y $800 \mu \mathrm{g} \mathrm{L}^{-1}$ presentaron una PM menor respecto al testigo y a la dosis de $1200 \mu \mathrm{g}$ $\mathrm{L}^{-1}$ (Cuadro 1C).

El 1-MCP puede reducir o no tener efecto sobre la PM de frutos de acuerdo con la especie. Osuna et al. (2005b) mencionan que el 1-MCP gaseoso disminuyó la PM de frutos de aguacate almacenados bajo condiciones de mercadeo (de 20 a $22^{\circ} \mathrm{C}$ ) mientras que en mango se reporta que el 1-MCP gaseoso no afectó la PM en frutos de la variedad Kent (Osuna, 2006). La disminución en PM de frutos de mango aplicados con 1-MCP puede ser debida al efecto que el 1-MCP tiene en la disminución de la respiración y la producción de etileno (Chaiprasart y Hansawasdi, 2009; Ortiz-Franco et al., 2016; Penchaiya et al., 2006; Watkins, 2006).

\section{Apariencia Externa}

El 1-MCP afectó negativamente la apariencia externa cuando se aplicó antes o después del THC. Los daños observados fueron pústulas en la cáscara y oscurecimiento de las lenticelas. En el experimento realizado en Nayarit en 2013 (Cuadro 2A) se observó que los frutos del testigo absoluto y del testigo 1-MCP no mostraron daño en ninguno de los muestreos realizados ya que sus valores estuvieron entre 0 y 1 ; sin embargo, al término de la simulación de traslado refrigerado y a madurez de consumo, los frutos del testigo con THC y los tratados con 1-MCP en cualquiera de las combinaciones con el THC, mostraron valores significativamente más altos, lo que provocó la pérdida de valor comercial. En contraste, para el experimento realizado en Florida (Cuadro 2B), al término de la simulación de traslado refrigerado, los frutos con la peor apariencia externa fueron aquellos del testigo THC y los de 1-MCP aplicados después de THC + hidroenfriado, los cuales alcanzaron los máximos valores de la escala; sin embargo, los tratamientos con 1-MCP aplicados antes o después del THC mostraron valores de apariencia externa de 1.4 y 2.0 (de buena a regular), pero a madurez de consumo ésta se mostró de regular a mala.

Cuando el efecto del THC y la dosis de 1-MCP fueron separados (experimento realizado en Nayarit en 2014), se observó que el THC fue el principal responsable de los daños en apariencia externa ya que en todos los muestreos los frutos con THC mostraron una diferencia significativa con respecto a aquEllos que no tuvieron THC, pues los primeros mostraron valores equivalentes a apariencia mala con pérdida de valor comercial, en tanto que los frutos sin $\mathrm{THC}$ tuvieron una apariencia de excelente a buena durante todo el desarrollo del experimento (Cuadro 2C). Por el contrario, en términos generales no se observaron diferencias significativas entre las dosis de 1-MCP, ya que los valores se mantuvieron dentro de la escala de apariencia externa buena a regular.

Los resultados aquí presentados evidencian que el THC afectó negativamente la apariencia de frutos de mango Keitt y no el tratamiento con 1-MCP (Figura 1). Es conocido que cuando los frutos son sometidos a factores externos de estrés como el THC se da lugar a una rápida generación de especies reactivas al oxígeno (superóxido, peróxido de hidrógeno, radicales de hidroxilo) potencialmente dañinas, las cuales interactúan con el sistema antioxidante de las plantas (Apel y Hirt, 2004). De acuerdo con Wang et al. (2009), el tratamiento con 1-MCP a frutos de mango inhibe la actividad de algunas de estas enzimas antioxidantes que incluyen a la catalasa, superóxido dismutasa y ascorbato peroxidasa, y disminuyen así los daños causados por el THC. También, autores como Cheng et al. (2012); Choi et al. (2008); Choi y Huber (2008) y Manganaris et al. (2008) mencionan que el 1-MCP acuoso no causa ningún daño externo cuando es aplicado a frutos de aguacate, tomate, ciruela o pera.

\section{Firmeza}

La formulación acuosa del 1-MCP mantuvo firmeza por 
Cuadro 2. Apariencia Externa de frutos de mango Keitt tratados con 1-MCP acuoso con o sin THC.

A) Experimento 1. Nayarit 2013.

\begin{tabular}{llccc}
\hline \multirow{2}{*}{ Tratamiento } & \multicolumn{4}{c}{ Etapas de muestreo } \\
\cline { 2 - 5 } & Inicial & 21 DR + 0 Amb & 21 DR + 4 Amb & 21 DR + 7 Amb \\
\hline 1. Testigo absoluto & $0.7 \mathrm{ab}$ & $0.7 \mathrm{~b}$ & $0.7 \mathrm{~b}$ & $0.4 \mathrm{c}$ \\
2. Testigo 1-MCP & $0.5 \mathrm{~b}$ & $0.4 \mathrm{~b}$ & $0.4 \mathrm{~b}$ & $0.2 \mathrm{c}$ \\
3. Testigo THC & $0.7 \mathrm{ab}$ & $2.4 \mathrm{a}$ & $2.4 \mathrm{a}$ & $1.5 \mathrm{ab}$ \\
4. 1-MCP antes THC & $1.1 \mathrm{a}$ & $2.7 \mathrm{a}$ & $2.0 \mathrm{a}$ & $1.2 \mathrm{~b}$ \\
5. 1-MCP después THC & $1.0 \mathrm{a}$ & $2.6 \mathrm{a}$ & $1.7 \mathrm{a}$ & $1.4 \mathrm{ab}$ \\
6. 1-MCP después THC + Hidro & $0.9 \mathrm{ab}$ & $2.6 \mathrm{a}$ & $1.9 \mathrm{a}$ & $2.1 \mathrm{a}$ \\
\hline
\end{tabular}

Medias con letras iguales dentro de columnas no son estadísticamente diferentes (Duncan, 0.05).

\section{B) Experimento 2. Florida 2013.}

\begin{tabular}{lccc}
\hline Tratamiento & \multicolumn{3}{c}{ Etapas de muestreo } \\
\cline { 2 - 4 } & Inicial & $21 \mathrm{DR}+0 \mathrm{Amb}$ & $21 \mathrm{DR}+4 \mathrm{Amb}$ \\
\hline 1. Testigo & $1.0 \mathrm{a}$ & $3.0 \mathrm{a}$ & - \\
2. 1-MCP después THC & $1.2 \mathrm{a}$ & $1.4 \mathrm{~b}$ & $2.6 \mathrm{a}$ \\
3. 1-MCP antes THC & $0.6 \mathrm{a}$ & $2.0 \mathrm{~b}$ & $3.0 \mathrm{a}$ \\
4. 1-MCP después THC + Hidro & $0.8 \mathrm{a}$ & $3.0 \mathrm{a}$ & - \\
\hline
\end{tabular}

Medias con letras iguales dentro de columnas no son estadísticamente diferentes (Duncan, 0.05).

C) Experimento 3. Nayarit 2014.

\begin{tabular}{|c|c|c|c|c|}
\hline \multirow{2}{*}{ Tratamiento } & \multicolumn{4}{|c|}{ Etapas de muestreo } \\
\hline & Inicial & $21 \mathrm{DR}+0 \mathrm{Amb}$ & $21 \mathrm{DR}+4 \mathrm{Amb}$ & $21 \mathrm{DR}+7 \mathrm{Amb}$ \\
\hline & \multicolumn{4}{|c|}{ Factor A (THC) } \\
\hline 1. $\operatorname{Sin}$ & $0.9 \mathrm{a}$ & $0.9 \mathrm{~b}$ & $0.2 b$ & $0.3 b$ \\
\hline \multirow[t]{2}{*}{ 2. Con } & $1.2 \mathrm{a}$ & $2.8 \mathrm{a}$ & $2.5 \mathrm{a}$ & $2.5 \mathrm{a}$ \\
\hline & \multicolumn{4}{|c|}{ Factor B (Dosis 1-MCP) } \\
\hline 1. $0 \mu \mathrm{g} \mathrm{L}^{-1}$ & $1.0 \mathrm{a}$ & $1.9 \mathrm{ab}$ & $1.2 b$ & $1.0 \mathrm{a}$ \\
\hline 2. $400 \mu \mathrm{g} \mathrm{L}^{-1}$ & $1.3 \mathrm{a}$ & $2.1 \mathrm{a}$ & $1.9 \mathrm{a}$ & $1.3 \mathrm{a}$ \\
\hline 3. $800 \mu \mathrm{g} \mathrm{L}^{-1}$ & $0.9 a$ & $1.6 \mathrm{~b}$ & $1.0 \mathrm{~b}$ & $1.3 \mathrm{a}$ \\
\hline 4. $1200 \mu g \mathrm{~L}^{-1}$ & $1.2 \mathrm{a}$ & $1.9 \mathrm{ab}$ & $1.4 \mathrm{ab}$ & $1.5 \mathrm{a}$ \\
\hline
\end{tabular}

Medias con letras iguales por factor dentro de columnas no son estadísticamente diferentes (Duncan, 0.05). Escala hedónica donde 0: Excelente; 1 : Buena; 2: Regular; 3: Mala.

mayor tiempo, particularmente cuando fue aplicada sin THC. Durante el experimento en Nayarit, 2013 (Cuadro 3A), al término de la simulación de traslado refrigerado, cualquiera de los frutos tratados con 1-MCP mantuvo mayor firmeza (83.7 a $92.0 \%$ ) que los del testigo absoluto o el testigo con THC, los cuales mantuvieron sólo el 75.7 y $78.2 \%$, respectivamente; sin embargo, al día 4 de la simulación de mercadeo los frutos tratados con 1-MCP sin THC mantuvieron $65 \%$ de la firmeza inicial y fueron significativamente superiores al resto de los tratamientos. Esta tendencia se mantuvo hasta madurez de consumo. En
Florida (Cuadro 3B), al término de la simulación de traslado refrigerado, el 1-MCP aplicado después del THC fue el mejor tratamiento al mantener $52.8 \%$ de la firmeza inicial, lo que favoreció significativamente al resto de los tratamientos; en madurez de consumo no se detectaron diferencias significativas en 1-MCP aplicado antes o después del THC. Cuando el efecto del THC y las dosis de 1-MCP fueron separados, no se detectaron diferencias significativas entre frutos con o $\sin \mathrm{THC}$ durante todas las etapas de muestreo (Cuadro $3 \mathrm{C}$ ), en tanto que para dosis de 1-MCP se detectaron ligeras diferencias entre tratamientos sin 
A) Experimento 1. Nayarit 2013.

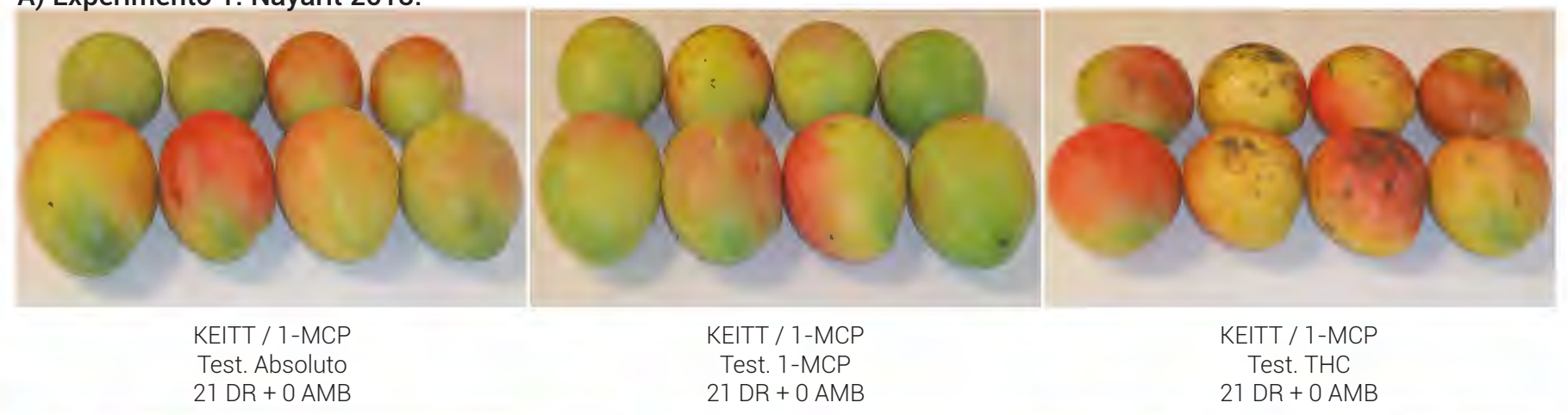

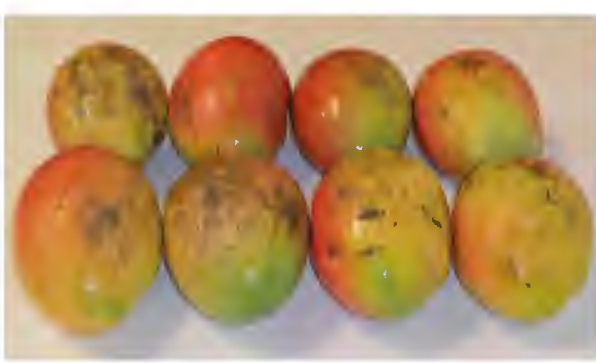

KEITT / 1-MCP

1-MCP antes THC

$21 \mathrm{DR}+0 \mathrm{AMB}$

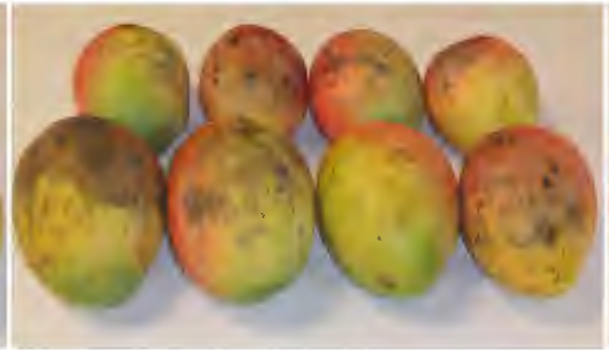

KEITT / 1-MCP

1-MCP después THC

$21 \mathrm{DR}+0 \mathrm{AMB}$

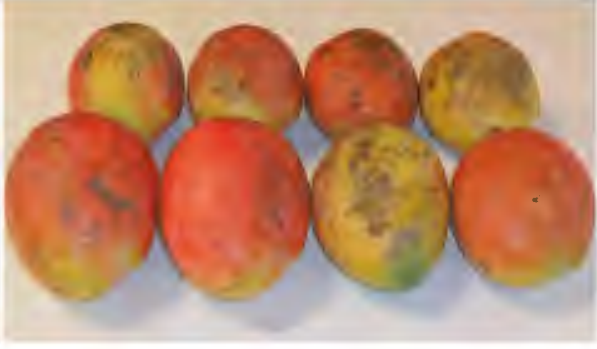

KEITT / 1-MCP

1-MCP después HIDROENF

$21 \mathrm{DR}+0 \mathrm{AMB}$

B) Experimento 2. Florida 2013.

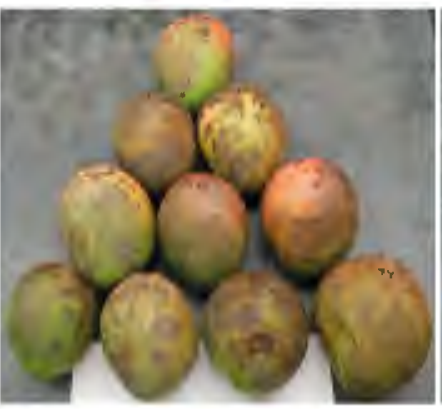

KEITT / CONTROL QHWT 21 DREF + 0 AMB

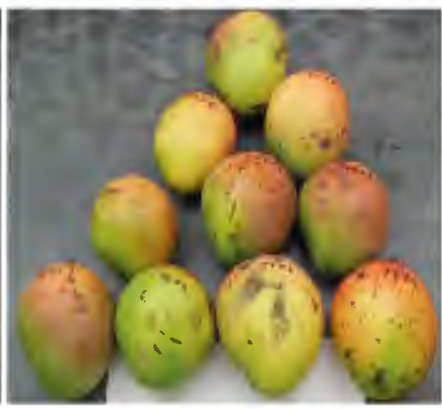

KEITT / 1-MCP AFTER QHWT 21 DREF + 0 AMB

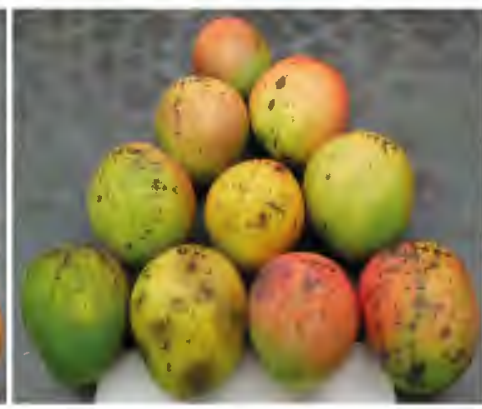

KEITT / 1-MCP BEFORE QHWT 21 DREF + 0 AMB

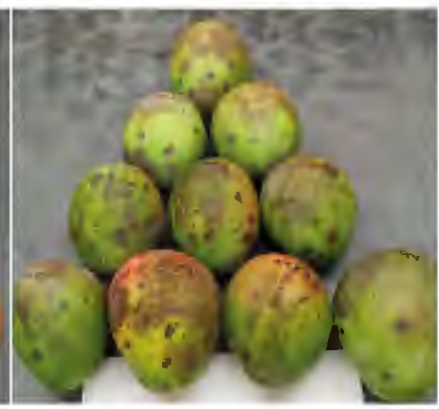

KEITT / 1-MCP AFTER QHWT + HYDROCOLLING 21 DREF + 0 AMB

Figura 1. Ilustración de la apariencia externa de los frutos de mango Keitt al término de la simulación de traslado refrigerado.

llegar a ser estadísticamente significativas. Estos resultados muestran que el 1-MCP acuoso mantuvo firmeza por mayor tiempo cuando fue aplicado sin THC, por lo que sólo podría ser recomendado para mercados que no demandan el THC obligatorio.

Algunos autores mencionan que el 1-MCP reduce la tasa de ablandamiento de frutos al inhibir la acción de las enzimas poligalacturonasa, pectin metilesterasa, pectato liasa y celulasa (Islas-Osuna et al., 2010; Muy et al., 2009; Razzaq et al., 2016). Osuna-García et al. (2007) evaluaron el efecto de 1-MCP a $300 \mathrm{ppb}$ después del tratamiento con agua caliente $\left(52{ }^{\circ} \mathrm{C}\right.$ por 5 min y $46.1^{\circ} \mathrm{C}$ por $110 \mathrm{~min}$ ), y reportaron que la acción del 1-MCP se redujo por el tiempo de tratamiento con agua caliente; a mayor tiempo de tratamiento hidrotérmico menor eficacia del 1-MCP; además, de acuerdo con Ngamchuachit et al. (2014), la formulación acuosa del 1-MCP disminuyó la tasa de ablandamiento de frutos Keitt, lo que resultó ser más eficiente cuando se aplicó antes del THC.

\section{Color de pulpa}

El efecto del 1-MCP acuoso no fue consistente en disminuir el desarrollo del color de pulpa. En Nayarit durante 2013 (Cuadro 4A), al término de la simulación de traslado 
C) Experimento 3. Nayarit 2014.

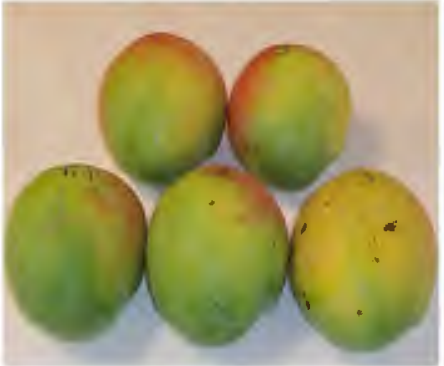

KEITT / 1-MCP

TESTIGO ABSOLUTO

21 DREF + 0 AMB

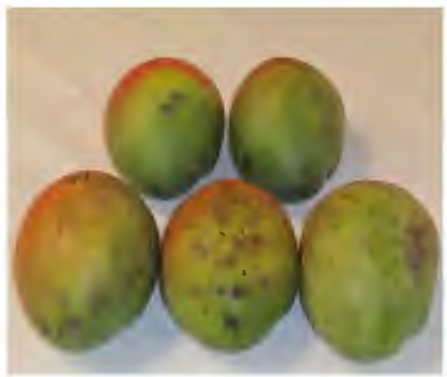

KEITT / 1-MCP

TESTIGO HIDROTÉRMICO

21 DREF + 0 AMB

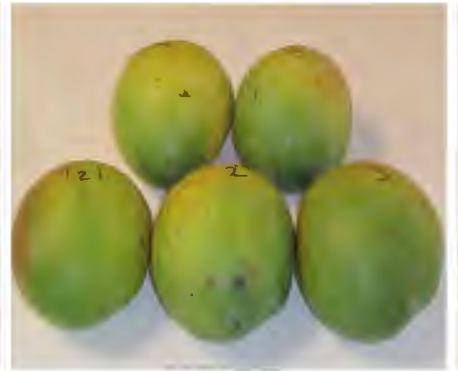

KEITT / 1-MCP

$400 \mu \mathrm{L} \mathrm{L}^{-1}$ SIN THC

$21 \mathrm{DR}+0 \mathrm{AMB}$

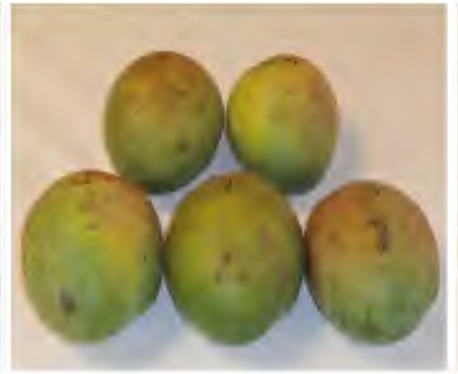

KEITT / 1-MCP

$400 \mu \mathrm{g} \mathrm{L}-1$ SIN THC

$21 \mathrm{DR}+0 \mathrm{AMB}$

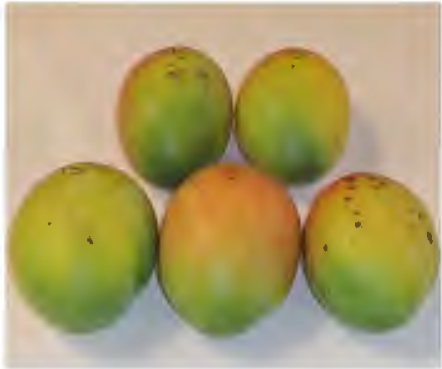

KEITT / 1-MCP

$800 \mu \mathrm{g} \mathrm{L}^{-1} \mathrm{SIN}$ THC

$21 \mathrm{DR}+0 \mathrm{AMB}$

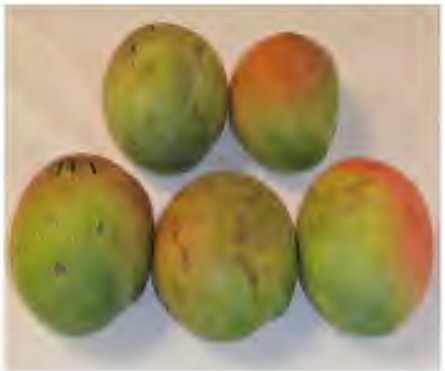

KEITT / 1-MCP

$800 \mu \mathrm{g} \mathrm{L}^{-1} \mathrm{CON}$ THC

$21 \mathrm{DR}+0 \mathrm{AMB}$

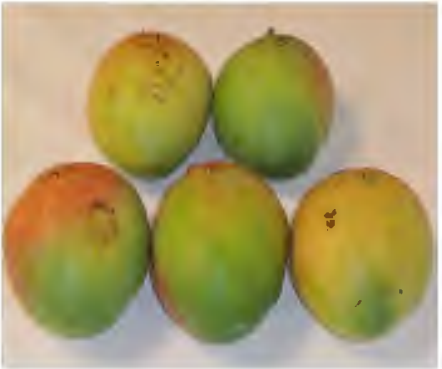

KEITT / 1-MCP

$1200 \mu \mathrm{g} \mathrm{L}-1$ SIN THC

$21 \mathrm{DR}+0 \mathrm{AMB}$

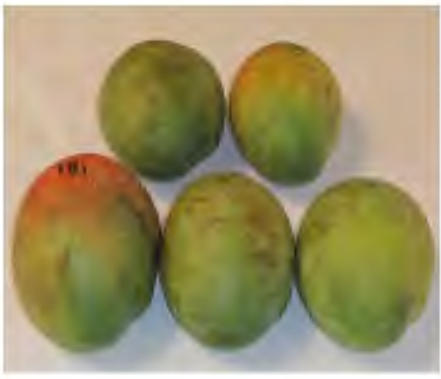

KEITT / 1-MCP

$1200 \mu \mathrm{g} \mathrm{L}^{-1}$ CON THC

$21 \mathrm{DR}+0 \mathrm{AMB}$

Figura 1. Continuación.

refrigerado, los frutos con mayor intensidad de color de pulpa ( ${ }^{\circ}$ hue $=83.2$ ) fueron los aplicados con el 1-MCP después del THC. El resultado fue estadísticamente diferente a todos los demás tratamientos ('hue de 86.0 a 89.4); sin embargo, durante la simulación de mercadeo y al momento del consumo fue el que mostró la menor velocidad de desarrollo del color de pulpa, y fue el testigo absoluto el que mostró mayor intensidad de color. En contraste, en Florida (Cuadro 4B), no se detectaron diferencias significativas para ninguno de los tratamientos en cualquiera de las etapas de muestreo.

Cuando el efecto del THC y las dosis de 1-MCP fueron separados, se encontraron diferencias significativas para frutos sin THC con respecto a frutos tratados con THC solo en el muestreo de 4 d en simulación de mercadeo, donde los frutos sin THC tuvieron una mayor intensidad de color (Cuadro 4C). Respecto al efecto de dosis de 1-MCP, sólo se detectaron diferencias significativas a madurez de consumo, la dosis más alta $\left(1200 \mu \mathrm{g} \mathrm{L} \mathrm{L}^{-1}\right)$ tuvo la menor intensidad de color de pulpa en comparación con el resto de tratamientos. Desafortunadamente hay poca literatura disponible para comparar el efecto del 1-MCP acuoso en frutos de mango; sin embargo, varios autores afirman que el 1-MCP gaseoso aplicado a frutos de Nam Dokmai, Namh-dawg-mai-sri-tong, Tommy Atkins y Apple Mango retrasó el pico climatérico y disminuyó la producción de etileno, lo que ayudó a mantener la firmeza y a retrasar los cambios de color de pulpa (Ambuko et al., 2013; Chaiprasart and Hansawasdi, 2009; Penchaiya et al., 2006).

\section{CONCLUSIONES}

La formulación acuosa del 1-MCP retrasó el proceso de maduración al mantener la firmeza por mayor tiempo, pero mostró una interacción negativa con el THC al causar manchas en la cáscara y oscurecimiento de lenticelas. El efecto negativo fue menor cuando el 1-MCP acuoso se aplicó antes o después del THC sin hidroenfriado y mayor cuando se aplicó después del THC + hidroenfriado. En contraste, el 1-MCP acuoso aplicado a frutos sin THC mantuvo $92 \%$ de la firmeza inicial al término de los $21 \mathrm{~d}$ de simulación de traslado refrigerado y retrasó hasta 4 d el proceso de maduración durante la simulación de mercadeo sin afectar la apariencia externa, por lo que puede ser una alternativa viable para mercados que no demandan el THC obligatorio. 
Cuadro 3. Firmeza (\% inicial) de frutos de mango Keitt tratados con 1-MCP acuoso con o sin THC.

A) Experimento 1. Nayarit 2013.

\begin{tabular}{lcccc}
\hline \multirow{2}{*}{ Tratamiento } & \multicolumn{4}{c}{ Etapas de muestreo } \\
\cline { 2 - 5 } 1. Testigo absoluto & Inicial & $21 \mathrm{DR}+0 \mathrm{Amb}$ & $21 \mathrm{DR}+4 \mathrm{Amb}$ & $21 \mathrm{DR}+7 \mathrm{Amb}$ \\
2. Testigo 1-MCP & $100.0 \mathrm{a}$ & $75.7 \mathrm{~b}$ & $24.7 \mathrm{~b}$ & $7.9 \mathrm{ab}$ \\
3. Testigo THC & $100.0 \mathrm{a}$ & $92.0 \mathrm{a}$ & $65.0 \mathrm{a}$ & $9.0 \mathrm{a}$ \\
4. 1-MCP antes THC & $100.0 \mathrm{a}$ & $78.2 \mathrm{~b}$ & $13.8 \mathrm{~b}$ & $5.5 \mathrm{c}$ \\
5. 1-MCP después THC & $100.0 \mathrm{a}$ & $91.6 \mathrm{a}$ & $15.6 \mathrm{~b}$ & $5.9 \mathrm{c}$ \\
6. 1-MCP después THC + Hidro & $100.0 \mathrm{a}$ & $83.7 \mathrm{ab}$ & $14.9 \mathrm{~b}$ & $6.3 \mathrm{bc}$ \\
\hline
\end{tabular}

Medias con letras iguales dentro de columnas no son estadísticamente diferentes (Duncan, 0.05).

B) Experimento 2. Florida 2013.

\begin{tabular}{lccc}
\hline \multirow{2}{*}{ Tratamiento } & \multicolumn{3}{c}{ Etapas de muestreo } \\
\cline { 2 - 4 } & Inicial & $21 \mathrm{DR}+0 \mathrm{Amb}$ & $21 \mathrm{DR}+4 \mathrm{Amb}$ \\
\hline 1. Testigo & $100.0 \mathrm{a}$ & $8.8 \mathrm{c}$ & - \\
2. 1-MCP después THC & $100.0 \mathrm{a}$ & $52.8 \mathrm{a}$ & $13.1 \mathrm{a}$ \\
3. 1-MCP antes THC & $100.0 \mathrm{a}$ & $34.2 \mathrm{~b}$ & $4.2 \mathrm{a}$ \\
4. 1-MCP después THC + Hidro & $100.0 \mathrm{a}$ & $18.5 \mathrm{c}$ & - \\
\hline
\end{tabular}

Medias con letras iguales dentro de columnas no son estadísticamente diferentes (Duncan, 0.05).

C) Experimento 3. Nayarit 2014.

\begin{tabular}{|c|c|c|c|c|}
\hline \multirow{2}{*}{ Tratamiento } & \multicolumn{4}{|c|}{ Etapas de muestreo } \\
\hline & Inicial & $21 \mathrm{DR}+0 \mathrm{Amb}$ & $21 \mathrm{DR}+4 \mathrm{Amb}$ & $21 \mathrm{DR}+7 \mathrm{Amb}$ \\
\hline & \multicolumn{4}{|c|}{ Factor $\mathrm{A}(\mathrm{THC})$} \\
\hline 1. $\operatorname{Sin}$ & $100.0 \mathrm{a}$ & $91.6 \mathrm{a}$ & 30.9 a & $21.4 \mathrm{a}$ \\
\hline \multirow[t]{2}{*}{ 2. Con } & $100.0 \mathrm{a}$ & $91.6 \mathrm{a}$ & $40.7 \mathrm{a}$ & $15.9 a$ \\
\hline & \multicolumn{4}{|c|}{ Factor B (Dosis 1-MCP) } \\
\hline 1. $0 \mu \mathrm{g} \mathrm{L}^{-1}$ & $100.0 \mathrm{a}$ & $95.6 \mathrm{a}$ & $26.8 \mathrm{a}$ & $8.1 \mathrm{a}$ \\
\hline 2. $400 \mu \mathrm{g} \mathrm{L}^{-1}$ & $100.0 \mathrm{a}$ & 89.3 a & $30.3 \mathrm{a}$ & $25.5 \mathrm{a}$ \\
\hline 3. $800 \mu \mathrm{g} \mathrm{L}^{-1}$ & $100.0 \mathrm{a}$ & $91.6 \mathrm{a}$ & $48.4 \mathrm{a}$ & $17.5 \mathrm{a}$ \\
\hline 4. $1200 \mu \mathrm{g} \mathrm{L}^{-1}$ & $100.0 \mathrm{a}$ & $89.7 \mathrm{a}$ & $37.8 \mathrm{a}$ & $22.5 \mathrm{a}$ \\
\hline
\end{tabular}

Medias con letras iguales por factor dentro de columnas no son estadísticamente diferentes (Duncan, 0.05). Firmeza inicial Nayarit $2013 \pm 300 \mathrm{~N}$; Florida $2013 \pm 150 \mathrm{~N}$; Nayarit $2014 \pm 280 \mathrm{~N}$.

\section{AGRADECIMIENTOS}

A Mauricio González de Agrofresh Inc., al National Mango Board, a la Universidad de Florida, a la Estación Experimental del USDA-ARS en Miami, FL, a las Empacadoras Agroservicios la 12 S. de R. L. de C. V. y Agroproductos de Nayarit S. P. R. de R. L.

\section{BIBLIOGRAFÍA}

Ambuko J., R. W. Githiga, M. J. Hutchnison, H. Gemma and W. O. Owino (2013) Effect of maturity stage and cultivar on the efficacy of 1-MCP treatments in mango fruits. Acta Horticulturae 1007:39-47.

AOAC, Association of Official Analytical Chemists (1984) Official Methods of Analysis. $14^{\text {th }}$ ed. Association of Official Analytical Chemists Inc. Arlington, VA. USA. $1006 \mathrm{p}$.

Apel K. and H. Hirt (2004) Reactive oxygen species: metabolism, oxidative stress, and signal transduction. Annual Review of Plant Biology 55:373-399.

Coêlho L. M. A., A. L. da Silva, S. S. Nunes A. and P. de Sá Santos (2006) Postharvest treatments with 1-methylcyclopropene in 'Tommy Atkins' mango fruit: effect of doses and number of applications. 
Cuadro 4. Color de pulpa ( $\left.{ }^{\circ} \mathrm{Hue}\right)$ de frutos de mango Keitt tratados con 1-MCP acuoso con o sin THC.

A) Experimento 1. Nayarit 2013.

\begin{tabular}{lcccc}
\hline \multirow{2}{*}{ Tratamiento } & \multicolumn{4}{c}{ Etapas de muestreo } \\
\cline { 2 - 5 } & Inicial & 21 DR + 0 Amb & $21 \mathrm{DR}+4 \mathrm{Amb}$ & $21 \mathrm{DR}+7 \mathrm{Amb}$ \\
\hline 1. Testigo absoluto & $93.6 \mathrm{a}$ & $89.4 \mathrm{a}$ & $70.8 \mathrm{~b}$ & $67.2 \mathrm{~b}$ \\
2. Testigo 1-MCP & $91.1 \mathrm{ab}$ & $89.0 \mathrm{a}$ & $76.3 \mathrm{ab}$ & $69.2 \mathrm{ab}$ \\
3. Testigo THC & $89.7 \mathrm{~b}$ & $86.0 \mathrm{ab}$ & $75.0 \mathrm{ab}$ & $68.9 \mathrm{ab}$ \\
4. 1-MCP antes THC & $91.0 \mathrm{ab}$ & $86.7 \mathrm{ab}$ & $71.6 \mathrm{ab}$ & $72.7 \mathrm{ab}$ \\
5. 1-MCP después THC & $92.6 \mathrm{a}$ & $83.2 \mathrm{~b}$ & $77.8 \mathrm{ab}$ & $73.7 \mathrm{a}$ \\
6. 1-MCP después THC + Hidro & $91.0 \mathrm{ab}$ & $87.4 \mathrm{ab}$ & $79.0 \mathrm{a}$ & $69.8 \mathrm{ab}$ \\
\hline
\end{tabular}

Medias con letras iguales dentro de columnas no son estadísticamente diferentes (Duncan, 0.05).

\section{B) Experimento 2. Florida 2013.}

\begin{tabular}{lccc}
\hline \multirow{2}{*}{ Tratamiento } & \multicolumn{3}{c}{ Etapas de muestreo } \\
\cline { 2 - 4 } & Inicial & $21 \mathrm{DR}+0 \mathrm{Amb}$ & $21 \mathrm{DR}+4 \mathrm{Amb}$ \\
\hline 1. Testigo & $84.9 \mathrm{a}$ & $84.6 \mathrm{a}$ & - \\
2. 1-MCP después THC & $84.3 \mathrm{a}$ & $82.5 \mathrm{a}$ & $83.4 \mathrm{a}$ \\
3. 1-MCP antes THC & $85.5 \mathrm{a}$ & $84.3 \mathrm{a}$ & $83.3 \mathrm{a}$ \\
4. 1-MCP después THC + Hidro & $85.6 \mathrm{a}$ & $85.3 \mathrm{a}$ & - \\
\hline
\end{tabular}

Medias con letras iguales dentro de columnas no son estadísticamente diferentes (Duncan, 0.05).

\section{C) Experimento 3. Nayarit 2014}

\begin{tabular}{|c|c|c|c|c|}
\hline \multirow{2}{*}{ Tratamiento } & \multicolumn{4}{|c|}{ Etapas de muestreo } \\
\hline & Inicial & $21 \mathrm{DR}+0 \mathrm{Amb}$ & $21 \mathrm{DR}+4 \mathrm{Amb}$ & $21 \mathrm{DR}+7 \mathrm{Amb}$ \\
\hline & \multicolumn{4}{|c|}{ Factor A (THC) } \\
\hline 1. $\operatorname{Sin}$ & $92.3 \mathrm{a}$ & $91.6 \mathrm{a}$ & $88.0 \mathrm{~b}$ & $87.1 \mathrm{a}$ \\
\hline \multirow[t]{2}{*}{ 2. Con } & $91.8 \mathrm{a}$ & $91.1 \mathrm{a}$ & $90.7 \mathrm{a}$ & $88.2 \mathrm{a}$ \\
\hline & \multicolumn{4}{|c|}{ Factor B (Dosis 1-MCP) } \\
\hline $1.0 \mu \mathrm{g} \mathrm{L}^{-1}$ & $92.0 \mathrm{a}$ & $90.3 \mathrm{a}$ & $88.0 \mathrm{a}$ & $86.2 b$ \\
\hline 2. $400 \mu \mathrm{g} \mathrm{L}^{-1}$ & $91.1 \mathrm{a}$ & $91.2 \mathrm{a}$ & $89.4 \mathrm{a}$ & $86.9 b$ \\
\hline 3. $800 \mu \mathrm{g} \mathrm{L}^{-1}$ & $92.5 a$ & $92.0 \mathrm{a}$ & $90.3 \mathrm{a}$ & $87.2 b$ \\
\hline 4. $1200 \mu \mathrm{g} \mathrm{L}^{-1}$ & $92.6 \mathrm{a}$ & $92.0 \mathrm{a}$ & $89.6 a$ & $89.6 a$ \\
\hline
\end{tabular}

Medias con letras iguales por factor dentro de columnas no son estadísticamente diferentes (Duncan, 0.05). ${ }^{+21}$ DR + 0, 4, 7 Amb: 21 d refrigeración; + 0, 4 o 7 d de simulación de mercadeo.

Revista Brasileira de Fruticultura 28:64-68.

Chaiprasart P. and C. Hansawasdi (2009) Effect of 1-methylcyclopropene on the shelf life of mango (Mangifera indica Linn.) cv. Nahmdawg-mai-sri-tong. Acta Horticulturae 820:725-730.

Cheng S., B. Wei and S. Ji (2012) A novel 1-methylcyclopropene treatment for quality control in Nangou pears at ambient temperature. African Journal of Agricultural Research 7:2236-2242.

Choi S. T. and D. J. Huber (2008) Influence of aqueous 1-methylcyclopropene concentration, immersion duration, and solution longevity on the postharvest ripening of breaker-turning tomato (Solanum lycopersicum L.) fruit. Postharvest Biology and Technology 49:147-154.

Choi S. T., P. Tsouvaltzis, C. I. Lim and D. J. Huber (2008) Suppression of ripening and induction of asynchronous ripening in tomato and avocado fruits subjected to complete or partial exposure to aqueous solutions of 1-methylcyclopropene. Postharvest Biology and Technology 48:206-214.

FAOSTAT, Food and Agriculture Organization Corporate Statistical Database (2011) World mango imports. Food and Agriculture Organization of the United Nations. Rome. http://www.fao.org/faostat/ es/\#home (Marzo 2015).

Islas-Osuna M. A., N. A. Stephens-Camacho, C. A. Contreras-Vergara, M. Rivera-Domínguez, E. Sánchez-Sánchez, M. A. Villegas-Ochoa and G. A. González-Aguilar (2010) Novel postharvest treatment reduces ascorbic acid losses in mango (Mangifera indica L.) var Kent. American Journal of Agricultural and Biological Sciences 5:342-349.

Manganaris G. A., C. H. Crisosto, V. Bremer and D. Holcroft (2008) Novel 1-methylcyclopropene immersion formulation extends shelf life of advanced maturity 'Joanna Red' plums (Prunus salicina 
Lindell). Postharvest Biology and Technology 47:429-433.

Muy R. D., B. Espinoza V., J. Siller C., J. A. Sañudo B., B. Valdez T. y T. Osuna E. (2009) Efecto del 1-metilciclopropeno (1-MCP) y de una película comestible sobre la actividad enzimática y calidad postcosecha del mango 'Ataulfo'. Revista Fitotecnia Mexicana 32:53-60.

Ngamchuachit P., D. M. Barret and E. J. Mitcham (2014) Effects of 1-methylcyclopropene and hot water quarantine treatment on quality of 'Keitt' mangos. Journal of Food Science 79:C505-C509.

Ortiz-Franco L. Z., A. Z. Ramírez-Villa, L. J. Cervantes-Mojica, M. D. MuyRangel, M. A. Gómez-Lim, H. S. García-Galindo, S. G. Sáyago-Ayerdi y E. Montalvo-González (2016) Efecto del 1-metilciclopropeno en la maduración de mango Ataulfo en condición simulada para exportación a Europa. Revista Fitotecnia Mexicana 39:305-316

Osuna G. J. A., J. A. Beltrán y V. Vázquez V. (2005a) Efecto del 1-metilciclopropeno (1-MCP) sobre el comportamiento postcosecha del aguacate 'Hass'. Revista Fitotecnia Mexicana 28:1-8.

Osuna G. J. A., J. A. Beltrán y M. A. Urías L. (2005b) Efecto del 1-metilciclopropeno (1-MCP) sobre la vida de anaquel y calidad de mango para exportación. Revista Fitotecnia Mexicana 28:271-278.

Osuna G. J. A. (2006) Validación semicomercial del SmartFresh ${ }^{\text {TM }}$ (1$\mathrm{MCP}$ ) en mango 'Kent' y 'Keitt' para exportación. In: Congreso de Ciencia y Tecnología Nayarit 2006. Tepic, Nayarit. pp:256-264.

Osuna-García J. A., I. Cáceres-Morales, E. Montalvo-González, M. Mata-Montes de Oca y B. Tovar-Gómez (2007) Efecto del 1-metilciclopropeno (1-MCP) y tratamiento hidrotérmico sobre la fisiología y calidad del mango 'Keitt'. Revista Chapingo Serie Horticultura 13:157-
163

Osuna-García J. A., M. H. Pérez-Barraza, V. Vázquez-Valdivia and J. A. Beltrán (2009) Methylcyclopropene (1-MCP), a new approach for exporting 'Kent' mangos to Europe and Japan. Acta Horticulturae 820:721-724.

Penchaiya P., R. Jansasithorn and S. Kanlavanarat (2006) Effect of 1-MCP on physiological changes in mango 'Nam Dokmai'. Acta Horticulturae 712:717-722

Pereira B. M., G. P. Pereira L., A. Rebouças S. J., F. Vianello and L. M. de Oliveira (2011) Post-harvest conservation of 'Tommy Atkins' mangoes treated with 1-methylcyclopropene. Revista Brasileira de Fruticultura 33:290-297.

Razzaq K., Z. Singh, A. S. Khan, S. A. K. U. Khan and S. Ullah (2016) Role of 1-MCP in regulating 'Kensington Pride' mango fruit softening and ripening. Plant Growth Regulation 78:401-411.

SAS Institute (2002) SAS/STAT User's Guide. Version 9. SAS Institute Inc. Cary, NC. pp: 1733-1906.

Wang B., J. Wang, X. Feng, L. Lin, Y. Zhao and W. Jiang (2009) Effects of 1-MCP and exogenous ethylene on fruit ripening and antioxidants in stored mango. Plant Growth Regulation 57:185-192.

Watkins C. B. (2006) 1-methylcyclopropene (1-MCP) based technologies for storage and shelf life extension. International Journal of Postharvest Technology and Innovation 1:62-68.

Watkins C. B. (2008) Overview of 1-methylcyclopropene trials and uses for edible horticultural crops. HortScience 43:86-94. 
